University of Nebraska - Lincoln

DigitalCommons@University of Nebraska - Lincoln

12-15-1997

\title{
Nanoscale imaging of domain dynamics and retention in ferroelectric thin films
}

Alexei Gruverman

University of Nebraska-Lincoln, agruverman2@unl.edu

H. Tokumoto

Joint Research Center for Atom Technology (JRCAT), Higashi 1-1-4, Tsukuba, Ibaraki 305, Japan

A. S. Prakash

University of Maryland, College Park

S. Aggarwal

University of Maryland, College Park

B. Yang

University of Maryland, College Park

See next page for additional authors

Follow this and additional works at: https://digitalcommons.unl.edu/physicsgruverman

Part of the Physics Commons

Gruverman, Alexei; Tokumoto, H.; Prakash, A. S.; Aggarwal, S.; Yang, B.; Wuttig, M.; Ramesh, R.; Auciello, O.; and Venkatesan, T., "Nanoscale imaging of domain dynamics and retention in ferroelectric thin films" (1997). Alexei Gruverman Publications. 3.

https://digitalcommons.unl.edu/physicsgruverman/3

This Article is brought to you for free and open access by the Research Papers in Physics and Astronomy at DigitalCommons@University of Nebraska - Lincoln. It has been accepted for inclusion in Alexei Gruverman Publications by an authorized administrator of DigitalCommons@University of Nebraska - Lincoln. 


\section{Authors}

Alexei Gruverman, H. Tokumoto, A. S. Prakash, S. Aggarwal, B. Yang, M. Wuttig, R. Ramesh, O. Auciello, and T. Venkatesan 


\title{
Nanoscale imaging of domain dynamics and retention in ferroelectric thin films
}

\author{
A. Gruverman and H. Tokumoto \\ Joint Research Center for Atom Technology (JRCAT), Higashi 1-1-4, Tsukuba, Ibaraki 305, Japan
}

A. S. Prakash, S. Aggarwal, B. Yang, M. Wuttig, and R. Ramesh ${ }^{\text {a) }}$

Department of Materials and Nuclear Engineering, University of Maryland, College Park, Maryland 20742

O. Auciello

Argonne National Laboratory, Materials Science Division, Argonne, Illinois 60439-4838

T. Venkatesan

Center for Superconductivity Research, University of Maryland, College Park, Maryland 20742

(Received 13 August 1997; accepted for publication 10 October 1997)

\begin{abstract}
We report results on the direct observation of the microscopic origins of backswitching in ferroelectric thin films. The piezoelectric response generated in the film by a biased atomic force microscope tip was used to obtain static and dynamic piezoelectric images of individual grains in a polycrystalline material. We demonstrate that polarization reversal occurs under no external field (i.e., loss of remanent polarization) via a dispersive continuous-time random walk process, identified by a stretched exponential decay of the remanent polarization. (c) 1997 American Institute of Physics. [S0003-6951(97)00550-0]
\end{abstract}

There is currently considerable technological interest worldwide in ferroelectric thin films for a large number of applications, including a new generation of nonvolatile memories with high-speed access and unlimited endurance. ${ }^{1}$

Fundamental issues in ferroelectric thin film capacitors are related to the exact nature of the complex domain structures and their interactions with defects, the domain dynamics under high-speed switching conditions, and their evolution in the absence of an external field (polarization relaxation). In this respect, theoretical models and electrical measurements (e.g., hysteresis loops and switching currents), have provided significant insight into understanding polarization switching in thin films. Polarization switching from one state to another under the influence of an external field has been studied extensively and is conventionally understood using classical nucleation and growth concepts of phase transformation (e.g., the Avrami model), as modified by Ishibashi. ${ }^{2}$ However, there have been very few studies focusing on the fundamental mechanisms that control the spontaneous reversal of polarization leading to a progressive loss of remanent polarization, a phenomenon referred to as retention loss. Application of high-resolution techniques such as SFM in conjunction with conventional electrical measurements provides a unique opportunity to achieve microscopic insight into the physical processes occurring in ferroelectric thin films. The recent progress in using SFM as an effective tool for high-resolution visualization of a domain structure in ferroelectric thin films provides the appropriate background for this letter. ${ }^{3-6}$

In a typical retention experiment, a capacitor is written into a polarization state and left in that state for fixed periods of time, after which the polarization remaining in the capacitor is measured, as illustrated, for example, in Fig. 1(a). ${ }^{7}$ In

\footnotetext{
a) Also at the Center for Superconductivity Research, University of Maryland, College Park, MD 20742.
}

such a macroscopic measurement, one obtains an average of the retention characteristics of an ensemble of grains. It is important to note that this reversal occurs in the absence of an external field and is driven primarily by internal depolarizing fields and elastic strain fields. In some cases the retention loss fits a log-time dependence; however in most cases it seems to fit a stretched exponential [i.e., equation of the form $\left.y=1-\exp \left(-k t^{n}\right)\right]$ better. In Fig. 1(b), the same data are plotted in a stretched exponential $\left[\ln \left(P / P_{i}\right)\right.$ time $]$ form with a very good fit and a slope of 0.24 . In a variety of systems, a stretched exponential behavior with $n<1$ has been characterized as a dispersive transport or random walk type process. ${ }^{8-10}$ Similarly, the good fit of the retention data to a stretched exponential type behavior suggests that this retention loss process occurs by a random walk process. However, these macroscopic studies do not provide information on the initial location of the reversal process and its progress as a function of time. In this respect, the SFM imaging method used in this study provides the capability for the direct observation of domain structure that is naturally linked to the polarization state of a ferroelectric film.

Experiments were carried out using the SFM/ piezoresponse method that is based on detection of the piezoelectric vibration of the ferroelectric sample. ${ }^{5}$ Application of the external voltage to the ferroelectric film results in a change of the film thickness due to the inverse piezoelectric effect. Whether the film thickness will expand or compress locally depends on its polarization state. It means that under the action of an ac electric field regions with opposite orientation of polarization will vibrate out of phase. The amplitude of the vibration signal provides information about the magnitude of the piezoelectric coefficient, while the polarization direction can be determined from the phase of the signal. ${ }^{11}$ The key point in the SFM piezoresponse mode is that the imaging ac field is applied between the bottom electrode and the conductive SFM tip, which is used as a movable top electrode, thus generating piezoelectric vibration in 

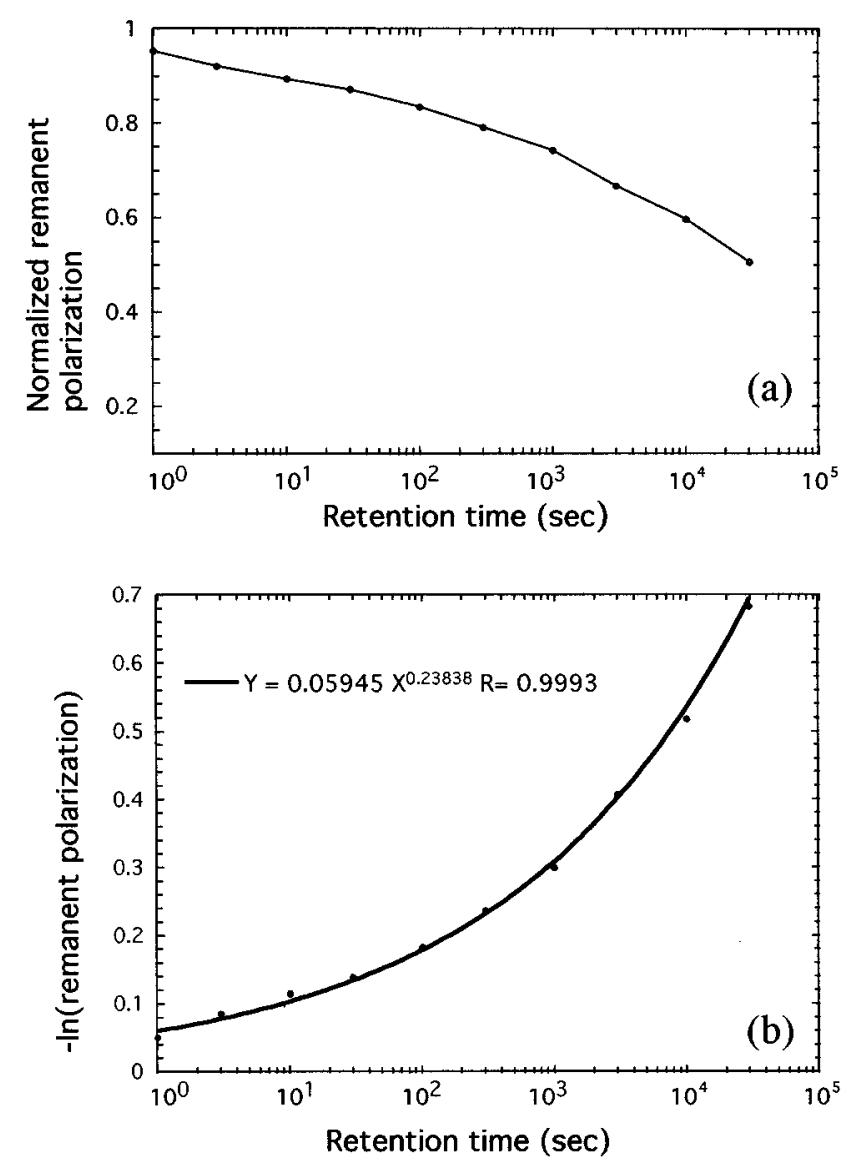

FIG. 1. Macroscopic retention experiments conducted electrically on polycrystalline PNZT using $5 \mathrm{~V}$ write pulses: (a) plot of switched polarization as function of log time and (b) plot of logarithm of switched polarization as function of time and depicting the stretched exponential fit.

a very small film area underneath the tip. Therefore a lateral resolution of the order of nanometers is achievable. Application of a dc voltage pulse to the probing tip generates a strong and sufficiently localized electric field, which makes possible nanoscale the writing of ferroelectric domains and the subsequent read-out.

A commercial force microscope (Seiko Instruments SPA 300) was employed in this study. In our experiments, piezoresponse images of the films were acquired in ambient air by applying a small ac voltage with an amplitude of $2.5 \mathrm{~V}$ (peak to peak) and a frequency of $10 \mathrm{kHz}$ while scanning the film surface. To apply the external voltage we used a standard gold coated $\mathrm{Si}_{3} \mathrm{~N}_{4}$ cantilever with a spring constant of 0.09 $\mathrm{N} / \mathrm{m}$. The probing tip, with an apex radius of about $20 \mathrm{~nm}$, was in mechanical contact with the uncoated film surface during the measurements. Cantilever vibration was detected using a conventional lock-in technique. A topographic image of the film surface was taken simultaneously with the domain imaging. Experiments were carried out using tetragonal $\mathrm{Pb}\left(\mathrm{Zr}_{0.2} \mathrm{Ti}_{0.8}\right) \mathrm{O}_{3}(\mathrm{PZT})$ films deposited by laser ablation onto Si substrates with a metal oxide electrode layer. Specifically, a PZT composition that showed a strong loss of remanent polarization with retention time, Fig. 1, was chosen for this study. Details of the thin film deposition protocols are described in earlier papers. ${ }^{12}$

Figures 2(a) and 2(b) show simultaneously acquired topographic and piezoresponse images of an as-deposited PZT
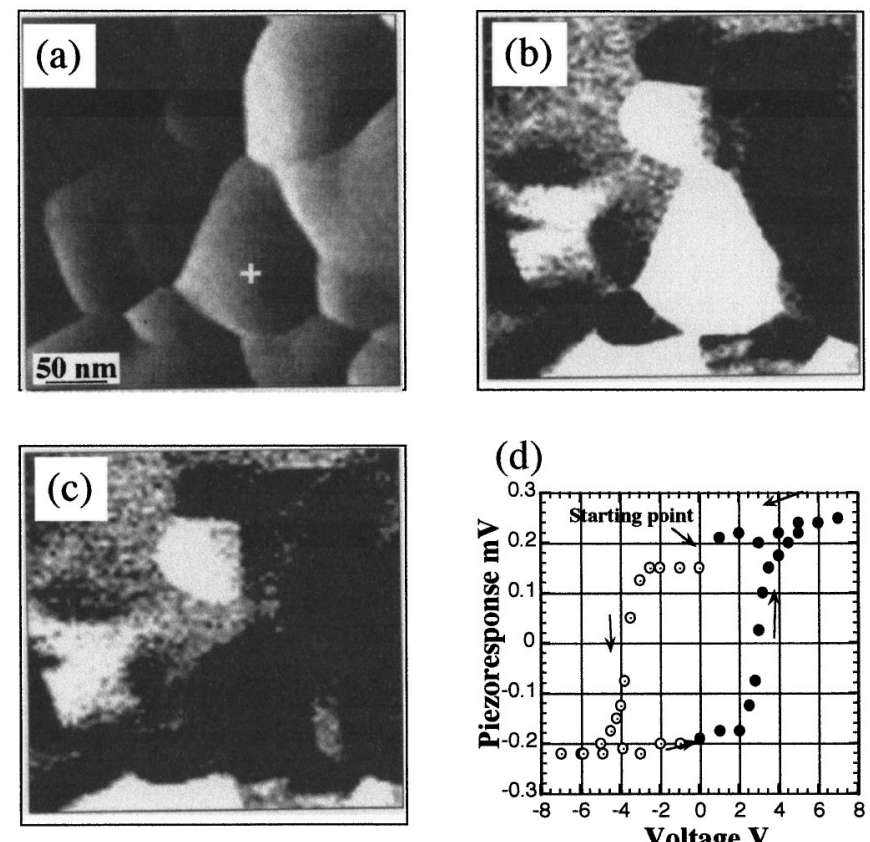

(d)

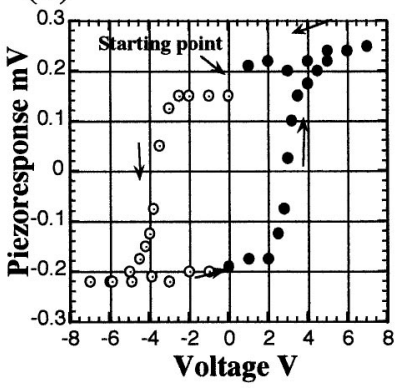

FIG. 2. Switching studies: (a) topography $\left(200 \times 200 \mathrm{~nm}^{2}\right)$, (b) piezoresponse of the as grown surface with $3 \mathrm{~V}$ ac, (c) piezoresponse after dc poling with $-6 \mathrm{~V}$, and (d) piezoelectric hysteresis loop.

20/80 film on a $\mathrm{La}_{0.5} \mathrm{Sr}_{0.5} \mathrm{CoO}_{3} / \mathrm{TiN} / \mathrm{Si}$ substrate (PZT 20/80-LCSO). By monitoring the phase of the piezoresponse signal it was determined that the bright regions in Fig. 2(b), which vibrate in phase with the ac imaging voltage, represent positive domains (polarization is towards the bottom electrode) while dark regions correspond to negative domains with the polarization vector oriented upward. To further substantiate this conclusion we measured the piezoresponse signal as a function of dc poling voltage. The probing tip was positioned at the center of a positively polarized grain where the voltage pulses were applied. After every pulse the piezoresponse signal was measured by applying a small ac voltage to the same point. The piezoresponse loop measurements were interrupted at the half-period to acquire the piezoresponse image of the grain under investigation. In Fig. 2(c), we can see that this grain of about $100 \mathrm{~nm}$ in size exhibits a reversed contrast compared to that in Fig. 2(b). Since the reversal of the contrast as well as the change in the sign of the piezoresponse signal [Fig. 2(d)] occurs under the negative voltage ("-" applied to the tip), it must be concluded that the grain was originally in the positive polarization state (polarization downward), which is consistent with the previous conclusion made from the phase monitoring of the film vibration. Contrast reversal in conjunction with the ferroelectric hysteresis behavior of the piezoresponse signal, shown in Fig. 2(d), is a clear proof of polarization reversal and demonstrates the ability of domain control at the nanometer scale. The size of the reversed domain depends essentially on the parameters of the switching voltage pulse. It will be shown below that using this technique it is possible to switch domains as small as $30 \mathrm{~nm}$ in diameter. It should be emphasized that the imaging process itself has no effect on the existing domain structure. Indeed, no change in the piezore- 

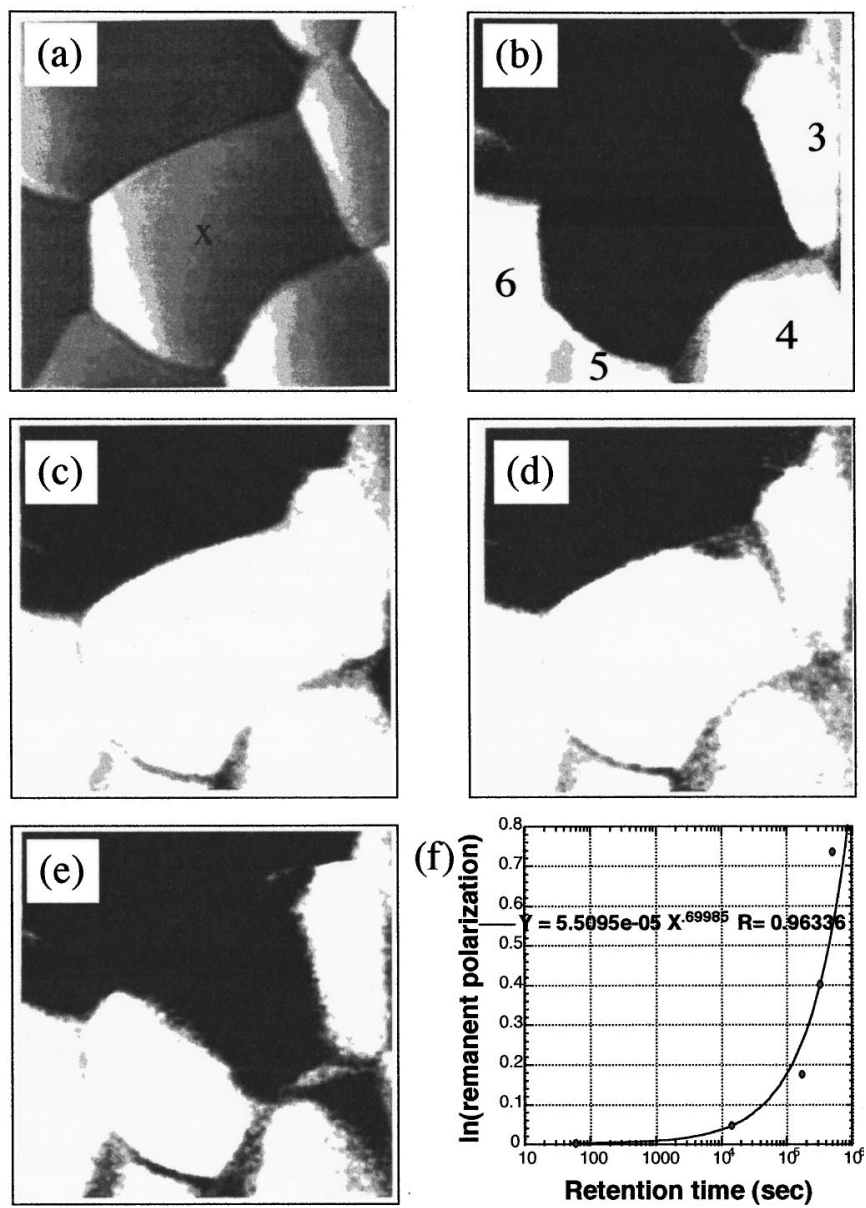

FIG. 3. SFM retention experiments conducted on PZT(20/80) grown on LSCO/TiN/Si using $6 \mathrm{~V}, 200 \mathrm{~ms}$ write pulse: (a) topography (200 $\times 200 \mathrm{~nm}^{2}$ ), (b) piezoresponse of the as grown surface with $2.3 \mathrm{~V} \mathrm{ac}$, (c) piezoresponse after dc poling grain 1 , (d) piezoresponse after $4 \mathrm{~h}$ with no external field, (e) piezoresponse after $140 \mathrm{~h}$, and (f) stretched exponential fit of the SFM studies plotted with the logarithm of the remanent switched grain area vs time.

sponse image is observed except on the region where poling voltages were applied.

Subsequently we investigated retention loss and the role of grain boundaries in the process of spontaneous backward (reverse) switching. The experimental sequence consisted of preliminary piezoresponse and topographic characterization of the film. The tip was then positioned at specific locations in a grain (at the center of the grain or at the grain periphery) polarized normally to the film surface and a single voltage pulse was applied. By varying the pulse width we could induce full or partial switching of the grain. Subsequently, piezoresponse images of the grain were recorded at various time intervals thus providing information about time evolution of the domain structure after the switching.

Figure 3(a) is a conventional topographic image of a single grain in the polycrystalline ensemble. For the sake of identification, the six grains (one central grain and five adjacent grains are numbered from 1 to 6). The piezoresponse in Fig. 3(b) shows uniform contrast across central grain 1, indicating that it is uniformly polarized in the negative direction (polarization upward). Upon application of a positive $6 \mathrm{~V}, 200 \mathrm{~ms}$ pulse to the center of the grain, it was fully switched into the opposite polarity, indicated by a change in grain contrast [Fig. 3(c)]. Note that the adjacent grains are still in their original polarization states. The piezoresponse images in Figs. 3(d) and 3(e) show time-evolution snapshots of the change in the domain structure of grain 1 that occurred after the removal of the dc field. Figure $3(d)$ is a piezoresponse image recorded $4 \mathrm{~h}$ after pulse switching; the first stages of backward switching, which started at the grain boundary, are discernible. This is direct evidence of the role played by grain boundaries in initiating spontaneous polarization reversal. In this case, the interesting point is that the reversal begins along the grain boundary that borders grain 2, suggesting that the electrostatic interaction across the grain boundary might influence the switching behavior. Once the reversal begins, it proceeds through the sidewise expansion of the reversed portion of the grain, as shown in Figs. 3(d) and 3(e). To summarize this backward switching process, in Fig. 3(f) the time dependence of the reversed fraction of the grain is fitted to a stretched exponential dependence with an exponent of 0.68. The difference in the exponent in this case compared to that of the macroscopic electrical measurements [Fig. 1(b)] is attributed to the different depolarizing fields and elastic fields in the microscopic measurements.

From the studies discussed here, it appears that the spontaneous reversal nucleates at the grain boundary and reversed domain stabilization requires a grain boundary. The data suggest that the spontaneous reversal may proceed via a random walk type mechanism similar to that postulated for magnetization reversal in spin glass. ${ }^{13}$ Our SFM studies fit a similar stretched exponential type behavior. The random walk type mechanism for polarization reversal brings forth new interpretations of the ferroelectric phenomenon and further studies are imperative to assess the validity of our suggested mechanism of polarization reversal.

The work at JRCAT is supported by the NEDO program and the work at the University of Maryland is supported by the NSF-MRSEC program.

${ }^{1}$ J. F. Scott and C. A. Araujo, Science 246, 1400 (1989).

${ }^{2}$ Y. Ishibashi, Ferroelectric Thin Films: Synthesis and Basic Properties (Gordon and Breach, Amsterdam, 1996), Vol. 10, p. 135.

${ }^{3}$ R. Luthi, H. Haefke, K. P. Meyer, E. Meyer, L. Howald, and H. J. Guntherodt, J. Appl. Phys. 74, 7461 (1993).

${ }^{4}$ K. Franke, J. Besold, W. Haessler, and C. Seegebarth, Surf. Sci. Lett. 302, L283 (1994).

${ }^{5}$ A. L. Gruverman, O. Auciello, and H. Tokumoto, Appl. Phys. Lett. 69, 3191 (1996)

${ }^{6}$ G. Zavala, J. H. Fendler, and S. T. McKinstry, J. Appl. Phys. 81, 7480 (1997).

${ }^{7}$ The test capacitor is $50 \mu \mathrm{m}$ in diameter, fabricated from a PZT thin film grown on a Si/poly-Si/TiN/Pt wafer with conductive oxide (LSCO) electrodes. In this experiment the capacitor was written with a short pulse (5 $\mathrm{V}, 8.6 \mu \mathrm{s})$ and after a specific period of time the polarization that is retained in the capacitor is measured using a $5 \mathrm{~V} 160 \mu$ s pulse.

${ }^{8}$ J. Kakalios, R. A. Street, and W. B. Jackson, Phys. Rev. Lett. 59, 1037 (1987)

${ }^{9}$ H. Scher and E. W. Montroll, Phys. Rev. B 12, 2455 (1975).

${ }^{10}$ K. L. Ngai, Comments Solid State Phys. 9, 127 (1979).

${ }^{11}$ K. Husimi and K. Kataoka, J. Appl. Phys. 29, 1247 (1958).

${ }^{12}$ O. Auciello, R. Dat, and R. Ramesh, in Ref. 2, p. 525.

${ }^{13}$ R. V. Chamberlain, G. Mozurkewich, and R. Orbach, Phys. Rev. Lett. 52, 867 (1984). 